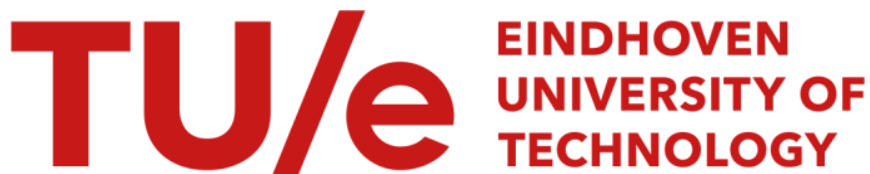

\section{New approach to orientationally disordered molecular crystals}

Citation for published version (APA):

Jansen, A. P. J. (1988). New approach to orientationally disordered molecular crystals. Journal of Chemical Physics, 88(3), 1914-1924. https://doi.org/10.1063/1.454115

DOI:

10.1063/1.454115

Document status and date:

Published: 01/01/1988

\section{Document Version:}

Publisher's PDF, also known as Version of Record (includes final page, issue and volume numbers)

\section{Please check the document version of this publication:}

- A submitted manuscript is the version of the article upon submission and before peer-review. There can be important differences between the submitted version and the official published version of record. People interested in the research are advised to contact the author for the final version of the publication, or visit the $\mathrm{DOI}$ to the publisher's website.

- The final author version and the galley proof are versions of the publication after peer review.

- The final published version features the final layout of the paper including the volume, issue and page numbers.

Link to publication

\section{General rights}

Copyright and moral rights for the publications made accessible in the public portal are retained by the authors and/or other copyright owners and it is a condition of accessing publications that users recognise and abide by the legal requirements associated with these rights.

- Users may download and print one copy of any publication from the public portal for the purpose of private study or research.

- You may not further distribute the material or use it for any profit-making activity or commercial gain

- You may freely distribute the URL identifying the publication in the public portal.

If the publication is distributed under the terms of Article 25fa of the Dutch Copyright Act, indicated by the "Taverne" license above, please follow below link for the End User Agreement:

www.tue.nl/taverne

Take down policy

If you believe that this document breaches copyright please contact us at:

openaccess@tue.nl

providing details and we will investigate your claim. 


\author{
A.P. J. Jansen \\ Institute of Theoretical Chemistry, University of Nijmegen, Toernooiveld, 6525 ED Nijmegen, \\ The Netherlands
}

(Received 24 July 1987; accepted 23 September 1987)

\begin{abstract}
A new theory is presented for the description of orientationally disordered molecular crystals. The theory is based on the thermodynamic variation principle with a generalized Ising Hamiltonian. The optimized single-molecule states are calculated, and the occupation of these states and the correlation in the occupation for pairs of molecules is determined via the cluster variation method. The theory is applied to the $\beta$ phase of solid nitrogen. A delocalized orientational probability distribution is found that is formed by six localized states which are equally occupied. Correlation functions for the orientations of nearest neighbors are given.
\end{abstract}

\section{INTRODUCTION}

Interest in orientationally disordered or "plastic" molecular crystals has been growing over the last years. This may be partially due to the fact that it is quite common for a molecular substance to have an orientationally disordered solid phase. Molecular crystals often show an order-disorder phase transition. The disordered phase at the higher temperatures is stabilized by a high entropy. In solid hydrogen the zero-point motions is so large that even at $T=0 \mathrm{~K}$ the crystal is orientationally disordered. ${ }^{1}$

The standard (quasi-)harmonic methods obviously do not work for the dynamics of these kinds of crystals. No other method has emerged yet that has been regarded widely as an appropriate method to describe the reorientations of the molecules. The most versatile (classical) method is molecular dynamics. A large number of orientationally disordered molecular crystals has been studied using this method, notably by Klein et al., ${ }^{2-8}$ Lynden-Bell et al. ${ }^{7-9}$ and Dove $e t$ $a l .{ }^{9,10}$ The major drawback of molecular dynamics is that it is a classical method. It fails where quantum effects are concerned. Furthermore, it is a simulation method, whereas one often prefers an analytical method in which the relations between various properties are more transparent.

The only quantum mechanical methods that have been used to our knowledge are the time independent and time dependent Hartree approximations, and the pseudo-spin method. The first two approximations are most frequently encountered in the form of a susceptibility approach (see the work of Michel) ${ }^{11-14}$ In our work we have usually called them mean field (MF) and random phase approximation (RPA). These approximations seem to work quite satisfactorily. However, as we have shown in the case of $\beta-\mathrm{N}_{2}$, there are crystals where a more sophisticated formalism is needed to treat the correlation in the occupation of different states. The pseudo-spin method attempts to do just that. ${ }^{15,16}$ This method however is only capable of handling two states per molecule. We will comment on all these methods later on.

The standard model for an order-disorder phase transition is the Ising model. ${ }^{17}$ In this paper we will show how to extend the Ising model so that it can be applied to molecular crystals. The main problem is what states to choose for the individual molecule. We will show how to calculate those states that yield the minimal free energy. In Sec. II A the method is outlined. In Sec. II B the cluster variation method for obtaining occupation numbers is presented. In Sec. III the results of the calculations on $\beta-\mathrm{N}_{2}$ and the $\alpha-\beta$ phase transition in solid nitrogen are presented. In Sec. IV the results are discussed, the method is compared to the methods mentioned above, and some improvements are suggested. The appendices serve to clarify some of the problems encountered in Sec. II.

\section{THEORY}

\section{A. Derivation of the method}

The method that we present in this paper is based on the thermodynamic variation principle or Gibbs-Bogoliubov inequality $^{18}$

$$
A_{\text {var }} \equiv A_{0}+\left\langle\hat{H}-\hat{H}_{0}\right\rangle_{0} \geqslant A,
$$

with

$$
\begin{aligned}
& A=-\beta^{-1} \ln \operatorname{Tr}\left[e^{-\beta \hat{H}}\right], \\
& A_{0}=-\beta^{-1} \ln \operatorname{Tr}\left[e^{-\beta \hat{H}_{0}}\right], \\
& \langle\hat{X}\rangle_{0}=\frac{1}{Z_{0}} \operatorname{Tr}\left[e^{-\beta \hat{H}_{0}} \hat{X}\right],
\end{aligned}
$$

and

$$
Z_{0}=\operatorname{Tr}\left[e^{-\beta \hat{H}_{0}}\right] .
$$

Here $\hat{H}$ is the exact Hamiltonian of the system, $A$ is the exact free energy, and $\widehat{X}$ is any operator. Carets are used to denote operators. The general form of the exact Hamiltonian can be written as

$$
\widehat{H}=\sum_{P} \hat{L}_{P}+\frac{1}{2} \sum_{P P} \hat{\Phi}_{P P^{\prime}},
$$

when we assume that there are no three- or more-particle interactions. The summation indices $P$ and $P^{\prime}$ denote the molecules in the crystal. The one-particle terms $\hat{L}_{P}$ contain the kinetic energy and possibly the crystal field. The twoparticle terms $\hat{\Phi}_{P P}$, contain the intermolecular interactions. As it is almost always impossible to calculate properties with the exact Hamiltonian $\hat{H}$, we use an approximate Hamiltonian $\widehat{H}_{0}$ for the calculations. The variational method consists of choosing a (parametrized) functional form for the Hamiltonian $\widehat{H}_{0}$. The "best" Hamiltonian $\widehat{H}_{0}$ is then obtained by 
varying it and thereby minimizing $A_{\mathrm{var}}$. If a sum of oneparticle operators is chosen for $\hat{H}_{0}$ then this yields the MF approximation. ${ }^{19,20}$ If a harmonic Hamiltonian is chosen for $\widehat{H}_{0}$ then this yields the self-consistent phonon (SCP) approximation. $^{21}$

We can write the exact Hamiltonian $\hat{H}$ in terms of an orthonormal basis of single-molecule states as

$$
\begin{aligned}
\hat{H}= & \sum_{P} \sum_{\alpha \beta}\left\langle\alpha(P)\left|\hat{L}_{P}\right| \beta(P)\right\rangle \widehat{E}_{\alpha \beta}^{(P)} \\
& +\frac{1}{2} \sum_{P P^{\prime}} \sum_{\alpha \beta \gamma \eta}\left\langle\alpha(P) \gamma\left(P^{\prime}\right)\left|\hat{\Phi}_{P P^{\prime}}\right| \beta(P) \eta\left(P^{\prime}\right)\right\rangle \\
& \times \widehat{E}_{\alpha \beta}^{(P)} \widehat{E}_{\left.\gamma \eta^{\prime}\right)}^{\left(P^{\prime}\right)}
\end{aligned}
$$

with

$$
\widehat{E}_{\alpha \beta}^{(P)} \equiv|\alpha(P)\rangle\langle\beta(P)| .
$$

The states $|\alpha(P)\rangle$ and $|\beta(P)\rangle$ are the states of the orthonormal basis.

In our method we approximate the Hamiltonian $\hat{H}$ by an operator $\widehat{H}_{0}$ which does not contain any nondiagonal terms; that is we write

$\hat{H}_{0}=\sum_{P} \sum_{\alpha} c_{P \alpha}^{(1)} \hat{E}_{\alpha \alpha}^{(P)}+\frac{1}{2} \sum_{P P} \sum_{\alpha \beta} c_{P \alpha, P^{\prime} \beta}^{(2)} \widehat{E}_{\alpha \alpha}^{(P)} \widehat{E}_{\beta \beta}^{\left(P^{\prime}\right)}$.

In the minimization of $A_{\text {var }}$ we vary both the coefficients $c_{P \alpha}^{(1)}$ and $c_{P \alpha, P^{\prime} \beta}^{(2)}$ and the basis of single-molecule states. If there are only two states per molecule then, apart from a constant, the Hamiltonian $\widehat{H}_{0}$ can be written as an classical Ising Hamiltonian

$$
\hat{H}_{\text {Ising }}=-\sum_{P P} J_{P P^{\prime}} \cdot \hat{\sigma}_{P z} \hat{\sigma}_{P^{\prime} z}+g \mu_{B} \sum_{P} H_{P} \hat{\sigma}_{P z}
$$

The Hamiltonian $\widehat{H}_{0}$ is thus a generalization of the Hamiltonian that is used in the pseudo-spin method. Our method can therefore be regarded as an extension of the pseudo-spin method. The Ising Hamiltonian and $\widehat{H}_{0}$ have the same properties. The eigenstates of $\hat{H}_{0}$ are product functions

$$
|\{\alpha\}|=\prod_{P} \otimes|\alpha(P)\rangle,
$$

just as for the Ising Hamiltonian. Its eigenvalues are

$$
E_{\{\alpha\}}=\sum_{P} \sum_{\alpha} c_{P \alpha_{P}}^{(1)}+\frac{1}{2} \sum_{P P^{\prime}} \sum_{\alpha \beta} c_{P \alpha_{P}, P^{\prime} \beta_{P}}^{(2)} .
$$

Consequently, the methods that have been devised for the Ising model to calculate thermodynamic averages $\langle\hat{X}\rangle_{0}$ may be used for $\widehat{H}_{0}$ as well.

To facilitate the variation of the basis states $\{|\alpha(P)\rangle\}$, we introduce a fixed orthonormal basis $\{|i(P)\rangle\}$ that is known. We define unitary matrices $U_{\alpha i}^{(P)}$ by

$$
|\alpha(P)\rangle=\sum_{i} U_{\alpha i}^{(P)}|i(P)\rangle
$$

Using this equation we can effect the variation of the basis $\{|\alpha(P)\rangle\}$ by varying the unitary matrices $U_{\alpha i}^{(P)}$. The basis $\{|i(P)\rangle\}$ will be called a reference basis. By substituting Eq. (11) into Eq. (7), and then substituting the resulting expression into Eq. (1), we obtain $A_{\mathrm{var}}$ as a function of the $c_{P \alpha}^{(1) \text { 's, }}$ the $c_{P \alpha, P^{\prime} \beta}^{(2)}$ 's and the $U_{\alpha i}^{(P)}$ 's. The functional form is intracta- ble however, because we obtain thermodynamic averages of the form $\langle\mid i(P)\rangle\langle j(P) \mid\rangle_{0}$ which depend on the $c_{P \alpha}^{(1)}$ 's, the $c_{P \alpha, P^{\prime} \beta}^{(2)}$ 's, and the $U_{\alpha i}^{(P)}$ 's in a very complicated way. Instead, we express $\widehat{H}_{0}$ and $\hat{H}$ in terms of the operators $\widehat{E}_{\alpha \beta}^{(P)}$. Equation (5) can be rewritten using the reference basis $\{|i(P)\rangle\}$ as follows:

$$
\begin{aligned}
\widehat{H}= & \sum_{P} \sum_{\alpha \beta} \hat{E}_{\alpha \beta}^{(P)} \sum_{i j} U_{\alpha i}^{(P) *}\left\langle i(P)\left|\hat{L}_{P}\right| j(P)\right\rangle U_{\beta j}^{(P)} \\
& +\frac{1}{2} \sum_{P P^{\prime}} \sum_{\alpha \beta \gamma \eta} \widehat{E}_{\alpha \beta}^{(P)} \widehat{E}_{\gamma \eta}^{\left(P^{\prime}\right)} \sum_{i j k l} U_{\alpha i}^{(P)^{*}} U_{\gamma k}^{\left(P^{\prime}\right) *} \\
& \times\left\langle i(P) k\left(P^{\prime}\right)\left|\hat{\Phi}_{P P^{\prime}}\right| j(P) l\left(P^{\prime}\right)\right\rangle U_{\beta j}^{(P)} U_{\eta l}^{\left(P^{\prime}\right)} .
\end{aligned}
$$

Substituting this equation, and Eq. (7), into Eq. (1) we obtain

$$
\begin{aligned}
A_{\mathrm{var}}= & A_{0}-\sum_{P} \sum_{\alpha}\left\langle\widehat{E}_{\alpha \alpha}^{(P)}\right\rangle_{0} \\
& \times\left[c_{P \alpha}^{(1)}-\sum_{i j} U_{\alpha i}^{(P)^{*}}\left\langle i(P)\left|\hat{L}_{P}\right| j(P)\right\rangle U_{\alpha j}^{(P)}\right] \\
& -\frac{1}{2} \sum_{P P^{\prime}} \sum_{\alpha \beta}\left\langle\widehat{E}_{\alpha \alpha}^{(P)} \hat{E}_{\beta \beta}^{\left(P^{\prime}\right)}\right\rangle_{0}\left[c_{P \alpha, P^{\prime} \beta}^{(2)}-\sum_{i j k l} U_{\alpha i}^{(P)^{*}} U_{\beta k}^{\left(P^{\prime}\right)^{*}}\right. \\
& \left.\times\left\langle i(P) k\left(P^{\prime}\right)\left|\hat{\Phi}_{P P^{\prime}}\right| j(P) l\left(P^{\prime}\right)\right\rangle U_{\alpha j}^{(P)} U_{\beta l}^{\left(P^{\prime}\right)}\right] .
\end{aligned}
$$

The traces hidden in this expression can be worked out using the states of Eq. (9). We then see that $A_{\text {var }}$ is again a function

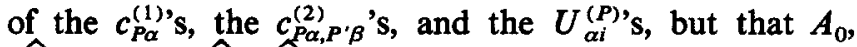
$\left\langle\widehat{E}_{\alpha \alpha}^{(P)}\right\rangle_{0}$, and $\left\langle\widehat{E}_{\alpha \alpha}^{(P)} \hat{E}_{\beta \beta}^{\left(P^{\prime}\right)}\right\rangle_{0}$ for all $P, P^{\prime}, \alpha$, and $\beta$ depend only

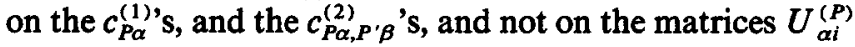
that change when varying the basis. In Eq. (13) only the matrices $U_{\alpha i}^{(P)}$ change that are explicitly shown. The thermodynamic averages $\left\langle\hat{E}_{\alpha \alpha}^{(P)}\right\rangle_{0}$ and $\left\langle\hat{E}_{\alpha \alpha}^{(P)} \widehat{E}_{\beta \beta}^{\left(P^{\prime}\right)}\right\rangle_{0}$ will appear frequently. We will call them one- and two-particle occupation numbers, respectively.

We minimize $A_{\text {var }}$ in the form of Eq. (13) by partial differentiation with respect to the $c_{P \alpha}^{(1)}$ 's, the $c_{P \alpha, P^{\prime} \beta}^{(2)}$ 's, and the $U_{\alpha i}^{(P)}$ 's. We have to remember that $A_{0}$ and the occupation

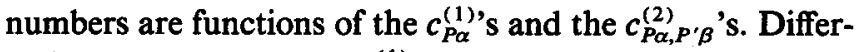
entiating with respect to $c_{P \alpha}^{(1)}$ yields

$$
\begin{aligned}
\frac{\partial A_{\text {var }}}{\partial c_{P \alpha}^{(1)}}= & \beta\left[\left\langle\hat{H}-\hat{H}_{0}\right\rangle_{0}\left\langle\hat{E}_{\alpha \alpha}^{(P)}\right\rangle_{0}\right. \\
& \left.-\left\langle\left(\hat{H}-\hat{H}_{0}\right) \hat{E}_{\alpha \alpha}^{(P)}\right\rangle_{0}\right],
\end{aligned}
$$

and with respect to $c_{P \alpha, P^{\prime} \beta}^{(2)}$ yields

$$
\begin{aligned}
\frac{\partial A_{\mathrm{var}}}{\partial c_{P \alpha, P^{\prime} \beta}^{(2)}}= & \beta\left[\left\langle\hat{H}-\widehat{H}_{0}\right\rangle_{0}\left\langle\widehat{E}_{\alpha \alpha}^{(P)} \widehat{E}_{\beta \beta}^{\left(P^{\prime}\right)}\right\rangle_{0}\right. \\
& \left.-\left\langle\left(\hat{H}-\hat{H}_{0}\right) \hat{E}_{\alpha \alpha}^{(P)} \widehat{E}_{\beta \beta}^{\left(P^{\prime}\right)}\right\rangle_{0}\right] .
\end{aligned}
$$

It is easy to see that for

$$
c_{P \alpha}^{(1)}=\left\langle\alpha(P)\left|\hat{L}_{P}\right| \alpha(P)\right\rangle
$$

and

$$
c_{P \alpha, P^{\prime} \beta}^{(2)}=\left\langle\alpha(P) \beta\left(P^{\prime}\right)\left|\widehat{\Phi}_{P P^{\prime}}\right| \alpha(P) \beta\left(P^{\prime}\right)\right\rangle
$$

the derivatives become zero. We can even prove that Eq. (15) will yield the absolute minimum of $A_{\text {var }}$ (see Appendix A). We note that $\langle\hat{H}\rangle_{0}=\left\langle\hat{H}_{0}\right\rangle_{0}$, in contradistinction to MF where they differ by $\left\langle\frac{1}{2} \Sigma_{P P^{\prime}}, \hat{\Phi}_{P P^{\prime}}\right\rangle_{0}$. 
Differentiating $A_{\text {var }}$ with respect to $U_{\alpha i}^{(P)}$ yields

$$
\begin{aligned}
\frac{\partial A_{\text {var }}}{\partial U_{\alpha i}^{(P)}}= & \left\langle\alpha(P)\left|\widehat{L}_{P}\right| i(P)\right\rangle\left\langle\hat{E}_{\alpha \alpha}^{(P)}\right\rangle_{0} \\
& +\sum_{P^{\prime}} \sum_{\beta}\left\langle\alpha(P) \beta\left(P^{\prime}\right)\left|\widehat{\Phi}_{P P^{\prime}}\right| i(P) \beta\left(P^{\prime}\right)\right\rangle \\
& \times\left\langle\widehat{E}_{\alpha \alpha}^{(P)} \hat{E}_{\beta \beta}^{\left(P^{\prime}\right)}\right\rangle_{0},
\end{aligned}
$$

and with respect to $U_{\alpha i}^{(P)^{*}}$ yields

$$
\begin{aligned}
\frac{\partial A_{\mathrm{var}}}{\partial U_{\alpha i}^{(P)^{*}}}= & \left\langle i(P)\left|\widehat{L}_{P}\right| \alpha(P)\right\rangle\left\langle\widehat{E}_{\alpha \alpha}^{(P)}\right\rangle_{0} \\
& +\sum_{P^{\prime}} \sum_{\beta}\left\langle i(P) \beta\left(P^{\prime}\right)\left|\hat{\Phi}_{P P^{\prime}}\right| \alpha(P) \beta\left(P^{\prime}\right)\right\rangle \\
& \times\left\langle\widehat{E}_{\alpha \alpha}^{(P)} \hat{E}_{\beta \beta}^{\left(P^{\prime}\right)}\right\rangle_{0} .
\end{aligned}
$$

These derivatives need not be zero, because the matrices $U_{\alpha i}^{(P)}$ must satisfy the restriction that they remain unitary. We introduce Lagrange multipliers $\lambda_{P \alpha \beta}$, and define

$$
\mathscr{A}=A_{\mathrm{var}}-\sum_{P} \sum_{\alpha \beta} \lambda_{P \alpha \beta} \sum_{i} U_{\alpha i}^{(P) *} U_{\beta i}^{(P)}
$$

The derivatives of $\mathscr{A}$, rather than of $A_{\text {var }}$, must be equated to zero. We thus find

$$
\begin{aligned}
\lambda_{P \beta \alpha}= & \left\langle\alpha(P)\left|\hat{L}_{P}\right| \beta(P)\right\rangle\left\langle\widehat{E}_{\alpha \alpha}^{(P)}\right\rangle_{0} \\
& +\sum_{P^{\prime}} \sum_{\gamma}\left\langle\alpha(P) \gamma\left(P^{\prime}\right)\left|\widehat{\Phi}_{P P^{\prime}}\right| \beta(P) \gamma\left(P^{\prime}\right)\right\rangle \\
& \times\left\langle\widehat{E}_{\alpha \alpha}^{(P)} \widehat{E}_{\gamma \gamma}^{\left(P^{\prime}\right)}\right\rangle_{0}
\end{aligned}
$$

and

$$
\begin{aligned}
\lambda_{P \alpha \beta}= & \left\langle\beta(P)\left|\widehat{L}_{P}\right| \alpha(P)\right\rangle\left\langle\widehat{E}_{\alpha \alpha}^{(P)}\right\rangle_{0} \\
& +\sum_{P^{\prime}} \sum_{\gamma}\left\langle\beta(P) \gamma\left(P^{\prime}\right)\left|\hat{\Phi}_{P P^{\prime}}\right| \alpha(P) \gamma\left(P^{\prime}\right)\right\rangle \\
& \times\left\langle\widehat{E}_{\alpha \alpha}^{(P)} \widehat{E}_{\gamma \gamma}^{\left(P^{\prime}\right)}\right\rangle_{0} .
\end{aligned}
$$

By taking the complex conjugate of Eq. (18a) we find

$$
\lambda_{P \alpha \beta}=\lambda_{P \beta \alpha}^{*} \text {. }
$$

Interchanging the indices $\alpha$ and $\beta$ in Eq. (18b) leads to

$$
\begin{aligned}
\left\langle\alpha(P)\left|\widehat{L}_{P}\right| \beta(P)\right\rangle\left\langle\widehat{E}_{\alpha \alpha}^{(P)}\right\rangle_{0}+\sum_{P^{\prime}} \sum_{\gamma}\left\langle\alpha(P) \gamma\left(P^{\prime}\right)\left|\widehat{\Phi}_{P P^{\prime}}\right|\right. \\
\times\left|\beta(P) \gamma\left(P^{\prime}\right)\right\rangle\left\langle\widehat{E}_{\alpha \alpha}^{(P)} \widehat{E}_{\gamma \gamma}^{\left(P^{\prime}\right)}\right\rangle_{0} \\
=\left\langle\alpha(P)\left|\hat{L}_{P}\right| \beta(P)\right\rangle\left\langle\widehat{E}_{\beta \beta}^{(P)}\right\rangle_{0} \\
\quad+\sum_{P^{\prime}} \sum_{\gamma}\left\langle\alpha(P) \gamma\left(P^{\prime}\right)\left|\widehat{\Phi}_{P P^{\prime}}\right| \beta(P) \gamma\left(\left(P^{\prime}\right)\right\rangle\right. \\
\quad \times\left\langle\widehat{E}_{\beta \beta}^{(P)} \widehat{E}_{\gamma \gamma}^{\left(P^{\prime}\right)}\right\rangle_{0} .
\end{aligned}
$$

This equation determines the basis $\{|\alpha(P)\rangle\}$. A method to solve it is shown in Appendix B. We only want to remark here that the method of Appendix B enables us also to determine whether the solution we obtain for Eq. (20) corresponds to a (local) minimum for $A_{\text {var }}$.

The "best" Hamiltonian $\hat{H}_{0}$ as given by Eq. (7) can be obtained by solving Eqs. (15) and (20). These equations are not independent. We solve both via an iterative procedure. We start by choosing a basis $\{|\alpha(P)\rangle\}$; then we calculate the

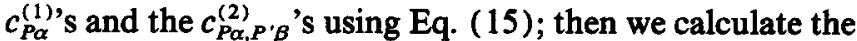
occupation numbers; and finally we solve Eq. (20). This gives us a new basis $\{\mid \alpha(P))\}$. If this new basis differs little from the old one, then we have obtained the "best" Hamiltonian $\hat{H}_{0}$. If the new basis differs too much then we repeat all calculations until convergence of the basis is reached. We have not yet addressed the problem of how to calculate the occupation numbers once numerical values for the $c_{P \alpha}^{(1)}$ 's and the $c_{P \alpha, P^{\prime} \beta}^{(2)}$ 's are known. Neither have we said how to calculate $A_{0}$. The next subsection will deal with these matters.

\section{B. The cluster variation method}

One has only been able to calculate the thermodynamic averages $\langle\widehat{X}\rangle_{0}$ for one- and some two-dimensional cases. ${ }^{22}$ The calculations of these thermodynamic averages pose what is called the Ising problem. Although few of these calculations have been done exactly, numerous approximation methods have been devised. ${ }^{23}$ This subsection will deal with one of them; the cluster variation method. ${ }^{24,25} \mathrm{We}$ have decided against using more accurate methods, because they would probably become too involved for our purpose. Simpler approximations cannot be used as they would yield results similar to MF. ${ }^{26}$ Furthermore, we have assumed that all molecules are equivalent. By this we mean that for any pair of molecules there is a symmetry operation of the system that relates the two molecules of the pair to each other. We then have to choose only the states of the basis $\{|\alpha(P)\rangle\}$ for one molecule. The states of all other molecules are determined by symmetry.

To simplify our notation we define

$$
x_{\alpha} \equiv\left\langle\widehat{E}_{\alpha \alpha}^{(Q)}\right\rangle_{0}
$$

and

$$
y_{\alpha \beta P} \equiv\left\langle\widehat{E}_{\alpha \alpha}^{(Q)} \widehat{E}_{\beta B}^{(P)}\right\rangle_{0} .
$$

Here $Q$ is a fixed molecule that is used for reference. The choice of $Q$ is arbitrary as all molecules are considered to be equivalent. We note that in general $y_{\alpha \beta P} \neq y_{\beta \alpha P}$. The $x_{\alpha}$ 's and the $y_{\alpha \beta P}$ 's are not independent. The following relations hold:

$$
\begin{aligned}
& \sum_{\alpha} x_{\alpha}=1, \\
& x_{\alpha}=\sum_{\beta} y_{\alpha \beta P},
\end{aligned}
$$

and

$$
x_{\alpha}=\sum_{\beta} y_{\beta \alpha P}
$$

Equations (23a) and (23b) are not independent, as $\Sigma_{\beta} y_{\alpha \beta P}$ $=x_{\alpha}$ for every $\alpha$, and $\Sigma_{\alpha} y_{\alpha \beta P}=x_{\beta}$ for every $\beta \neq 1$, implies $\Sigma_{\alpha} y_{\alpha 1 P}=x_{1}$.

The cluster variation method tries to answer the following question: "Given all one-, two-, and more-particle occupation numbers, what is the free energy?" It is fairly easy to write down an expression for the energy. The problem is the entropy. For a linear chain we can use the cluster variation method for the Ising model without modifications (see Refs. 24 and 25). We derive an expression for the entropy of an ensemble of $M$ linear chains. The total number of ways an 
ensemble of $M$ linear chains with given occupation numbers $x_{a}$ and $y_{\alpha \beta P}$ can be constructed if there are $N$ molecules per chain and if we ignore end effects is

$$
G=\left[\frac{\Pi_{\alpha}\left(M x_{\alpha}\right) !}{\Pi_{\alpha \beta}\left(M y_{\alpha \beta P}\right) !}\right]^{N} \text {. }
$$

The entropy for the ensemble is given by

$$
S=k \ln G \text {. }
$$

Equation (24) yields the exact entropy if there are only nearest-neighbor interactions. ${ }^{24,25}$ No method has been found yet to determine $G$ for an ensemble of two- or three-dimensional systems. Following Refs. 24 and 25 an approximation for $G$ can be obtained by multiplying the right-hand side of Eq. (24) by a correction factor

$$
\Gamma=\left[\frac{\Pi_{\alpha}\left(M x_{\alpha}\right) !}{\Pi_{\alpha \beta}\left(M y_{\alpha \beta P}\right) !}\right] /\left[\frac{M !}{\Pi_{\alpha}\left(M x_{\alpha}\right) !}\right]
$$

for different nearest neighbors. The introduction of $\Gamma$ is called the Bethe approximation. ${ }^{24,25}$ The expression for $G$ becomes

$$
G=\left[\frac{\left(\Pi_{\alpha}\left(M x_{\alpha}\right) !\right)^{z-1}}{\left(\Pi_{P} \Pi_{\alpha \beta}\left(M y_{\alpha \beta P}\right) !\right)^{1 / 2}(M !)^{(1 / 2) z-1}}\right]^{N},
$$

with $z$ the number of nearest neighbors. Using Stirling's approximation for the factorials we find for the entropy per molecule

$$
S=k(z-1) \sum_{\alpha} x_{\alpha} \ln x_{\alpha}-\frac{1}{2} k \sum_{P} \sum_{\alpha \beta} y_{\alpha \beta P} \ln y_{\alpha \beta P} .
$$

The expression for the energy per molecule is

$$
E=\sum_{\alpha} c_{Q \alpha}^{(1)} x_{\alpha}+\frac{1}{2} \sum_{P} \sum_{\alpha \beta} c_{Q \alpha, P \beta}^{(2)} y_{\alpha \beta P}
$$

With these expressions we can determine the occupation numbers $x_{\alpha}$ and $y_{\alpha \beta P}$ by minimizing the free energy $A=E-T S$.

We perform the minimization procedure again by differentiating. Because of the relations Eqs. (22) and (23) we introduce Lagrange multipliers $\lambda, \mu_{P \alpha}$, and $v_{P \alpha}$. We define

$$
\begin{aligned}
\mathscr{A} \equiv & A-\lambda \sum_{\alpha} x_{\alpha}-\sum_{P} \sum_{\alpha} \mu_{P \alpha}\left(x_{\alpha}-\sum_{\beta} y_{\alpha \beta P}\right) \\
& -\sum_{P} \sum_{\alpha \neq 1} v_{P \alpha}\left(x_{\alpha}-\sum_{\beta} y_{\beta \alpha P}\right) .
\end{aligned}
$$

The derivatives of $\mathscr{A}$ with respect to $x_{\alpha}$ and $y_{\alpha \beta P}$ for all $P, \alpha$, and $\beta$ must be zero. Solving the resulting equations we find

$$
x_{\alpha}=\exp \left[-1+\tilde{c}_{\alpha}^{(1)}+\tilde{\lambda}+\sum_{P}\left(\tilde{\mu}_{P \alpha}+\tilde{v}_{P \alpha}\right)\right]
$$

and

$$
\begin{aligned}
y_{\alpha \beta P}= & \exp \left[-1+\tilde{c}_{\alpha \beta P}^{(2)}\right. \\
& \left.+2(z-1) \tilde{\mu}_{P_{\alpha}}+2(z-1) \tilde{v}_{P \alpha}\right],
\end{aligned}
$$

with

$$
\tilde{c}_{\alpha}^{(1)}=\frac{c_{Q \alpha}^{(1)}}{k T(z-1)},
$$

$$
\begin{aligned}
& \tilde{c}_{\alpha \beta P}^{(2)}=-\frac{1}{k T} c_{Q \alpha, P \beta}^{(2)}, \\
& \tilde{\lambda}=-\frac{\lambda}{k T(z-1)}, \\
& \tilde{\mu}_{P \alpha}=-\frac{\mu_{P \alpha}}{k T(z-1)},
\end{aligned}
$$

and

$$
\tilde{\boldsymbol{v}}_{P \alpha}=-\frac{v_{P \alpha}}{k T(z-1)} .
$$

Here $k$ denotes the Boltzmann constant. In writing down these equations we have put $v_{P 1}=0$. The Lagrange multipliers can be obtained by substituting Eq. (31) in Eqs. (22) and (23). We then get the following set of nonlinear equations:

$$
\begin{gathered}
\tilde{\lambda}+\ln \sum_{\alpha} \exp \left[\tilde{c}_{\alpha}^{(1)}+\sum_{P}\left(\tilde{\mu}_{P \alpha}+\tilde{v}_{P \alpha}\right)\right]=1, \\
\tilde{\lambda}-2(z-1) \tilde{\mu}_{P \alpha}+\tilde{c}_{\alpha}^{(1)}+\sum_{P^{\prime}}\left(\tilde{\mu}_{P^{\prime} \alpha}+\tilde{v}_{P^{\prime} \alpha}\right) \\
-\ln \sum_{\beta} \exp \left[\tilde{c}_{\alpha \beta P}^{(2)}+2(z-1) \tilde{v}_{P \beta}\right]=0, \\
\tilde{\lambda}-2(z-1) \tilde{v}_{P \alpha}+\tilde{c}_{\alpha}^{(1)}+\sum_{P^{\prime}}\left(\tilde{\mu}_{P^{\prime} \alpha}+\tilde{v}_{P^{\prime} \alpha}\right) \\
-\ln \sum_{\beta} \exp \left[\tilde{c}_{\alpha \beta P}^{(2)}+2(z-1) \tilde{\mu}_{P \beta}\right]=0 .
\end{gathered}
$$

These equations can be solved numerically via the NewtonRaphson method. ${ }^{27}$ Equation (31) can then be used to obtain the occupation numbers. The energy and entropy are obtained via Eqs. (28) and (29). As a final remark we want to point out that if $c_{Q \alpha, P \beta}^{(2)}=c_{Q \beta, P \alpha}^{(2)}$ for all $\alpha$ and $\beta$, for a certain $P$, then $y_{\alpha \beta P}=y_{\beta \alpha P}$. For suppose $y_{\alpha \beta P} \neq y_{\beta \alpha P}$ for some $\alpha$ and $\beta$, then the substitution $y_{\alpha \beta P} \rightarrow \frac{1}{2}\left(y_{\alpha \beta P}+y_{\beta \alpha P}\right)$ will yield the same energy, and a larger entropy, and hence a lower free energy. However, in general we have $y_{\alpha \beta P} \neq y_{\beta \alpha P}$.

\section{RESULTS}

We have applied the method described in the previous section to the librational motions in $\beta-N_{2}$. At low pressures this phase exists between $T=35.6 \mathrm{~K}$ and $T=63.1 \mathrm{~K}$. At elevated pressures this phase exists even at temperatures as high as room temperature. ${ }^{28}$ The space group is $P 6_{3} / m m c$, and there are two molecules per unit cell (see Fig. 1) ${ }^{29-31} \mathrm{X}$ ray diffraction experiments have shown no preferred orientation for the molecules. There only seems to be a slight preference for an angle of $56^{\circ}$ between the molecular axis and the crystallographic $c$ axis. Different models have been contrived to account for the $x$-ray ${ }^{29-31}$ and neutron ${ }^{32}$ diffraction measurements, and also for the nuclear magnetic resonance/ nuclear quadrupole resonance experiments. ${ }^{33}$ One model assumes that the molecules process freely or only slightly hindered around the $c$ axis. ${ }^{29-32,34}$ Another model assumes that the molecules "choose" at random one out of a number of fixed orientations. ${ }^{29-31}$ And a third model assumes jumps between these orientations. ${ }^{35}$

In a previous paper we have reported the results of MF 


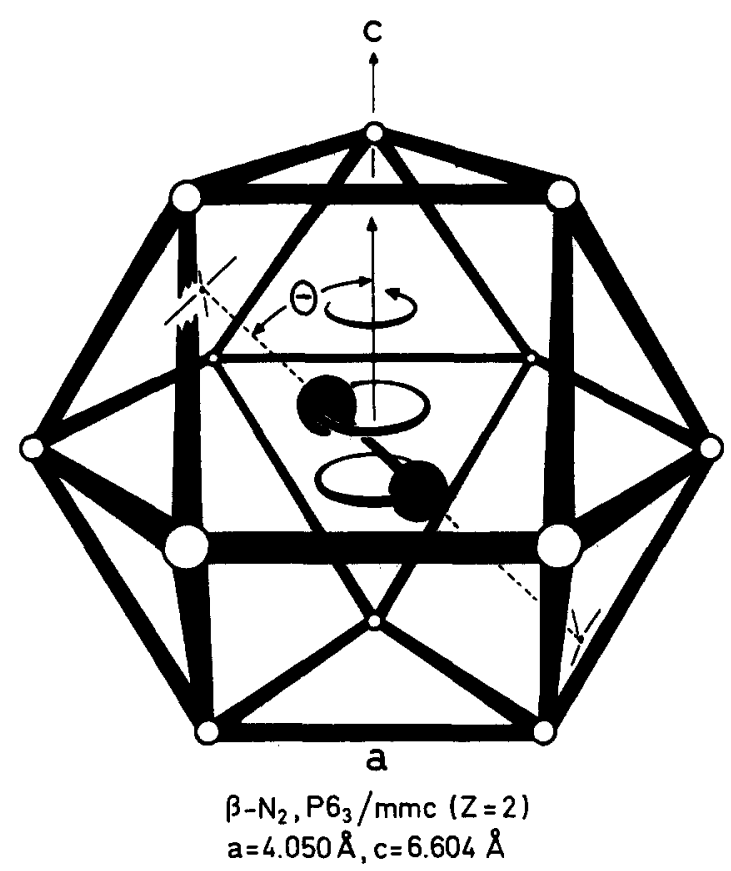

FIG. 1. The structure of $\beta-N_{2}$.

calculations on the librations in $\beta-\mathrm{N}_{2} \cdot{ }^{35} \mathrm{We}$ found two possible orientational probability distributions for the molecules. If the local reference frames of the two molecules in the unit cell were chosen to be identical, then a disordered phase with delocalized orientations of the molecules was found (see Fig. 2 of Ref. 35). If the local reference frames of the two molecules in the unit cell were allowed to rotate with respect to each other, then an ordered phase with localized orientations of the molecules was found (see Fig. 3 of Ref. 35). The orientations of the two molecules in the unit cell were found to be rotated by $180^{\circ}$ about the $c$ axis. The ordered phase was about $0.87 \mathrm{~kJ} \mathrm{~mol}^{-1}$ lower in energy at $T=0 \mathrm{~K}$, but had also a much lower entropy. At high temperatures (above $T=120 \mathrm{~K}$ ) the ordered phase showed an order-disorder transition to the disordered phase. Neither of the phases however, showed a transition to the $\alpha$ phase at any reasonable temperature. For all temperatures at which the MF calculations were done the $\alpha$ phase had a lower free energy. The results of the MF calculations were thus incorrect in two aspects: the molecules were found to have a preferred orientation in the $\beta$ phase, and no $\alpha-\beta$ phase transition was found. We therefore introduced the following ad hoc hypothesis. There are six localized orientations that are solutions of MF. These six orientations can be transformed into each other by rotations of multiples of $60^{\circ}$ around the $c$ axis. We assumed that there are free jumps between these six orientations. These free jumps yielded a delocalized picture for the orientations of the molecules. They also yielded an extra $k \ln 6$ for the entropy. This lowered the free energy of the $\beta$ phase so much that a phase transition to the $\alpha$ phase at $T=35 \mathrm{~K}$ was found. A theoretical foundation for the hypothesis could not be given, however.

As our new method is more complicated than the MF calculations we have had to limit the number of states and the number of molecules in the lattice sums. We have used

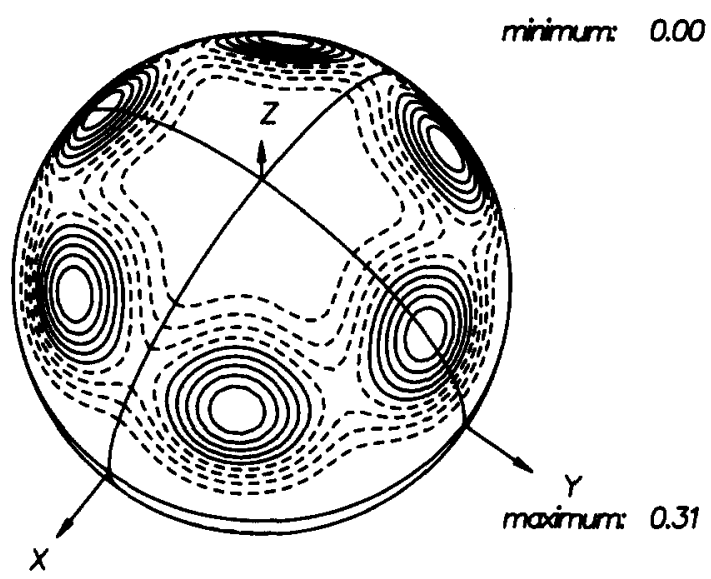

FIG. 2. Orientational probability distribution of $\beta-\mathrm{N}_{2}$ according to the new method for $T>30 \mathrm{~K}$. The dashed contours denote a low probability; the solid contours denote a high probability. Subsequent contours differ by $10 \%$ of the maximum of the probability distribution.

six states $|\alpha(P)\rangle$ per molecule in most of our calculations, and only interactions with the 12 nearest neighbors have been taken into account. But already in this "small" calculation a set of 133 nonlinear equations has to be solved in order to obtain the occupation numbers [see Eq. (33) ]. Furthermore, we have assumed that all molecules are equivalent. We thus need only states for one molecule in the calculations. The states of the other molecules can be obtained via (proper) rotations. Equations (15a) and (20) have to be solved for only one molecule, and the right-hand side of Eq. (15b) has only to be calculated for one molecule and its nearest neighbors. In Appendix B, Eq. (B6) must be augmented with the restriction that $X_{i j}^{(P)}=X_{i j}^{\left(P^{\prime}\right)}$ for all $i, j, P$, and $P^{\prime}$. Even with the restriction that all molecules must be equivalent there are various possibilities for the number of sublattices and their configuration. We have found however, that the sublattices that yield the ordered phase in the MF calculations (see above) give the lowest free energy at low temperature. At high temperatures it becomes irrelevant how the sublattices are chosen (see below). The results that are presented in this paper have been obtained with the intermolecular potential of Berns and van der Avoird. ${ }^{36}$ We have also done some calculations with the newer $a b$ initio potential of van der Avoird et al. ${ }^{37}$ The results of these calculations, however, do not differ qualitatively from those obtained with the Berns-van der Avoird potential. We have therefore done the calculations with the simpler potential.

We have started the calculations by choosing states for our reference basis $\{\mid i(P))\}$. As the calculations can only be done with a limited number of states some care must be taken in choosing these states. Although the states $|\alpha(P)\rangle$ are improved in the iterative procedure that has been described in the paragraph following Eq. (20), one has to bear in mind that one is restricted to the space that is spanned by the states $|i(P)\rangle$. The final states will always be linear combinations of the states of the reference basis $\{|i(P)\rangle\}$. For the calculations on $\beta-\mathrm{N}_{2}$ we have chosen a reference basis that might yield ordered as well as disordered phases. We have calculated the localized MF ground state at $T=0 \mathrm{~K}$ in a basis of spherical harmonics $Y_{l, m}$ with $l_{\max }=10$ (see Ref. 35). From 
this state we have generated five other states by five subsequent rotations of $60^{\circ}$ around the $c$ axis. The six states thus obtained we have orthogonalized. These orthogonal states form our reference basis $\{|i(P)\rangle\}$. The final states $|\alpha(P)\rangle$ can be localized or delocalized. If the calculations yield localized states of which only one is occupied then we have obtained an ordered phase. A disordered phase results when the localized states are equally occupied or when the final states are delocalized.

At low temperatures we have found an ordered phase with only one of the six initial states appreciably occupied. The other initial states are mixed to yield new but only slightly occupied states. The orientational probability distribution resembles closely that of Fig. 3 of Ref. 35. The phase is thus almost identical to the ordered phase of MF. Above $T=30$
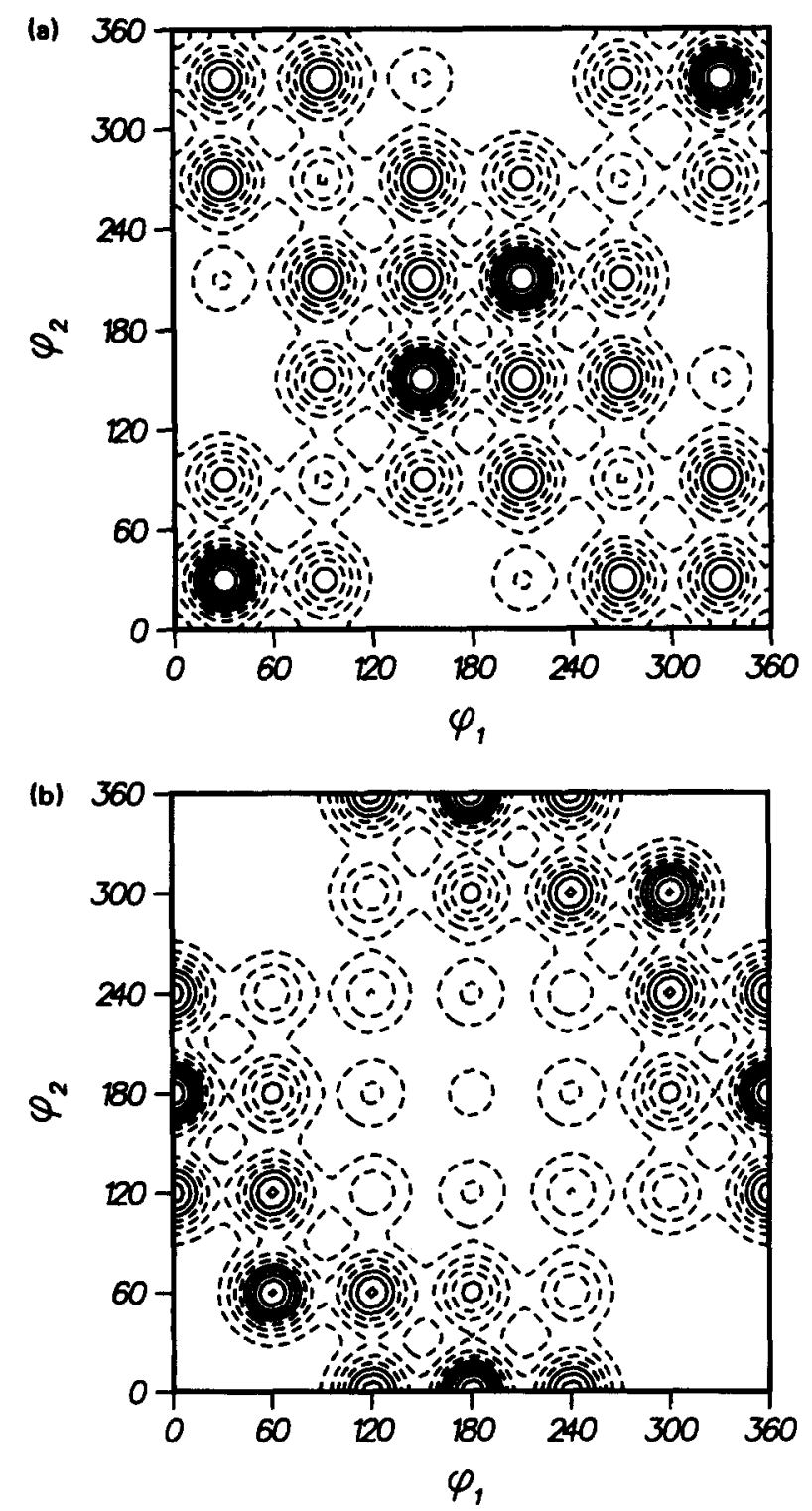

FIG. 3. Correlation function as given by Eq. (36) with $\theta_{1}=\theta_{2}=55^{\circ}$. The reference frame is oriented with the $z$ axis along the crystallographic $c$ axis, and the $x$ axis along the projection on the $a b$ plane of the intermolecular vector $\mathbf{R}_{12}=\mathbf{R}_{\mathbf{2}}-\mathbf{R}_{\mathbf{1}}$. Solid contours denote a high value of the correlation function, and dashed contours denote a low value. Subsequent contours differ by $10 \%$ of the maximum of the correlation function. The correlation function for in-plane nearest neighbors (a) and nearest neighbors in adjacent layers with molecule 2 higher than molecule 1 (b) are shown.
$\mathrm{K}$ this ordered phase becomes unstable. Instead we have found a disordered phase (see Fig. 2). The states of this phase are the initial states which have become equally occupied.

This is of course radically different from MF where no stable disordered phase has been found below $T=120 \mathrm{~K}$. Whereas the (unstable) disordered phase in MF has a high energy, the disordered phase in the new method has a very low energy. At $T=30 \mathrm{~K}$ the energy of the disordered phase is even lower than the energy of the ordered phase at $T=0$ $\mathrm{K}$. The reason for this difference is that there is a very large correlation in the occupation of the various states for nearest neighbors, i.e.,

$$
\left\langle\widehat{E}_{\alpha \alpha}^{(Q)} \widehat{E}_{\beta \beta}^{(P)}\right\rangle_{0} \neq\left\langle\widehat{E}_{\alpha \alpha}^{(Q)}\right\rangle_{0}\left\langle\widehat{E}_{\beta \beta}^{(P)}\right\rangle_{0},
$$

while in MF the left- and right-hand side of Eq. (34) are intrinsically equal. We can define the orientational correlation function for a pair of molecules 1 and 2 ,

$$
\begin{aligned}
& \rho\left(\Omega_{1} ; \Omega_{2}\right) \\
& \quad=\frac{\Sigma_{n} \exp \left(-\beta E_{n}\right) \int d \Omega_{3} \cdots d \Omega_{N}\left|\psi_{n}\left(\Omega_{1} ; \ldots ; \Omega_{N}\right)\right|^{2}}{\Sigma_{n} \exp \left(-\beta E_{n}\right)},
\end{aligned}
$$

with $\Omega_{i}=\left(\theta_{i}, \phi_{i}\right)$ and $d \Omega_{i}=\sin \theta_{i} d \theta_{i} d \phi_{i}$. The summation in this equation is over the exact eigenstates of the Hamiltonian $\hat{H}$. In our model we approximate this function by

$$
\rho\left(\Omega_{1} ; \Omega_{2}\right) \approx \sum_{\alpha \beta}\left\langle\widehat{E}_{\alpha \alpha}^{(1)} \widehat{E}_{\beta \beta}^{(2)}\right\rangle_{0}\left|\psi_{\alpha}\left(\theta_{1}, \phi_{1}\right)\right|^{2}\left|\psi_{\beta}\left(\theta_{2}, \phi_{2}\right)\right|^{2} .
$$

The functions $\psi_{\alpha}$ correspond to the states $|\alpha(P)\rangle$. A contour plot of this function with $\theta_{1}=\theta_{2}=55^{\circ}$, which corresponds to the maximum of the orientational probability distribution, is shown in Fig. 3. We see that in the new method nearest neighbors within the $a b$ plane prefer to be parallel to each other, but at the same time not perpendicular to the line that connects their centers of gravity. In the preferred orientations the molecules are only a few degrees from the absolute minimum of the intermolecular potential. The preferred orientations of the nearest neighbors in adjacent layers, with respect to each other, vary. The difference of $\phi_{1}$ and $\phi_{2}$ ranges from $0^{\circ}$ to $360^{\circ}$.

The free energy of $\beta-\mathrm{N}_{2}$ as a function of temperature is shown in Fig. 4. We note that there is an unphysical discontinuity at $T=30 \mathrm{~K}$. This discontinuity is caused by the cluster variation method. A close inspection of the expression for the free energy that results from Eqs. (28) and (29) reveals that the free energy has two different minima at low temperature. One of them corresponds to the ordered phase. The other corresponds to the disordered phase. The free energy of the disordered phase is lower also at low temperatures. However, below $T=26 \mathrm{~K}$ the entropy of the disordered phase, Eq. (28), becomes negative. We recall that in the derivation of Eq. (28) we introduced a correction [see Eq. (26) ]. It is easy to see that this correction yields a negative contribution to the entropy. If the correction becomes too large then the total entropy can become negative. It is obvious that when the entropy becomes negative the cluster variation method can no longer be used. Unfortunately, it is 


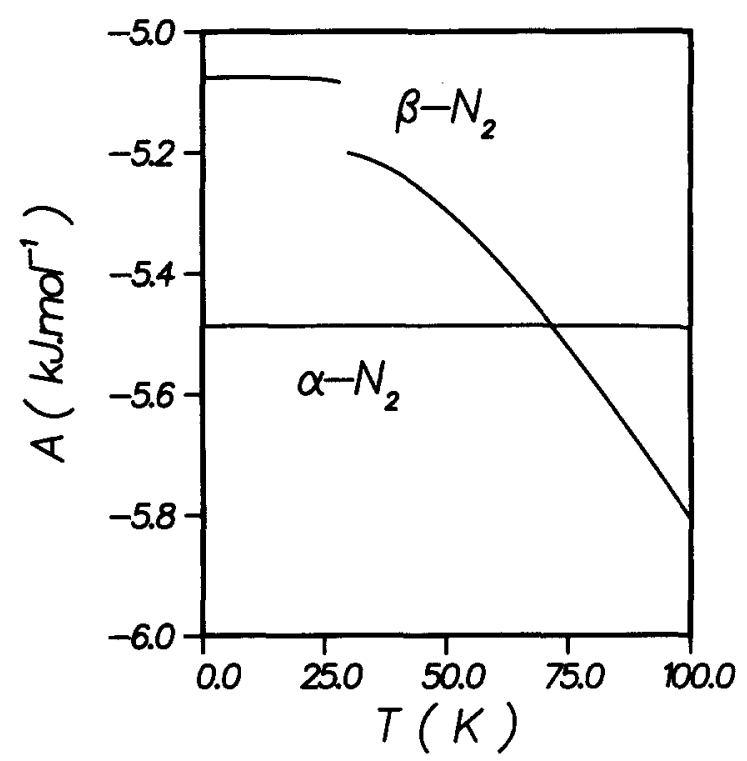

FIG. 4. Free energy of $\alpha$ - and $\beta-\mathrm{N}_{2}$ calculated using Eqs. (28) and (29).

not clear what the free energy of the phases would be if better expressions were available. It might be that the disordered phase is the stabler one also at temperatures below $30 \mathrm{~K}$ but that is by no means certain.

Also shown in Fig. 4 is the free energy of $\alpha-\mathrm{N}_{2}$ as calculated with the new method. The calculations have been performed again using the Berns-van der Avoird potential and only nearest neighbors interactions. Four states have been used. These states have been obtained in the same way as the states in $\beta-\mathrm{N}_{2}$. The only difference is that rotations of $90^{\circ}$ around the $c$ axis (instead of $60^{\circ}$ ) have been used. We note that the free energy curve is an almost horizontal line. The molecules are very localized. Even at high temperatures only one of the states is occupied. The new method predicts an $\alpha-$ $\beta$ phase transition at about $T_{\alpha \beta}=72 \mathrm{~K}$, which is quite reasonable, given the approximations made in the calculations (e.g., only nearest-neighbors interactions). One might argue that this phase transition is rather artificial. As the molecules in the $\alpha$ phase are localized a better (i.e., lower) estimate for the free energy would be obtained when some of the lower excited MF states would be included in the calculations. This is undoubtedly the case. However, inclusion of excited MF states would also lower the free energy of $\beta-N_{2}$. We think therefore, that as long as calculations with a large number of states is unfeasible, that it is best of perform the calculations with similar states.

The one-particle properties in the disordered phase in $\beta$ $\mathrm{N}_{2}$ do not change with temperature. This is a consequence of the fact that the occupation $\left\langle\widehat{E}_{\alpha \alpha}^{(P)}\right\rangle_{0}$ of the states does not change with temperature. In order to study the temperature dependence of the orientational probability distribution we have done calculations including the spherical harmonic $Y_{0,0}$ as an extra state. With this spherical harmonic the orientational probability distribution can become broader. The thermodynamic averages of some tesseral harmonics are shown in Table I. We note that the results seem to be somewhat better than the MF results, although due to the
TABLE I. Orientational form factors $\left(S_{l, m}\right)$ of $\beta-N_{2}$. The $S_{l, m}$ 's are real linear combinations of spherical harmonics."

\begin{tabular}{|c|c|c|c|c|}
\hline \multirow[b]{3}{*}{$T=$} & \multicolumn{3}{|c|}{ Theory } & \multirow{3}{*}{$\begin{array}{c}\text { Experiment } \\
\text { Ref. } 32 \\
30 \mathrm{~K}\end{array}$} \\
\hline & \multicolumn{2}{|c|}{ MF, Ref. 35} & \multirow[b]{2}{*}{$\begin{array}{c}\text { This paper } \\
30 \mathrm{~K}\end{array}$} & \\
\hline & $\begin{array}{c}\text { Delocalized } \\
30 \mathrm{~K}\end{array}$ & $\begin{array}{l}\text { Localized } \\
30 \mathrm{~K}\end{array}$ & & \\
\hline$\left\langle S_{2,0}\right\rangle$ & -0.027 & 0.048 & 0.042 & $\cdots$ \\
\hline$\left\langle S_{4,0}\right\rangle$ & -0.082 & -0.233 & -0.244 & $\cdots$ \\
\hline$\left\langle S_{6,0}\right\rangle$ & 0.029 & 0.042 & 0.057 & $\cdots$ \\
\hline$\left\langle S_{6,6}\right\rangle$ & -0.016 & -0.035 & -0.062 & $\cdots$ \\
\hline$T=$ & $55 \mathrm{~K}$ & $55 \mathrm{~K}$ & $55 \mathrm{~K}$ & $55 \mathrm{~K}$ \\
\hline$\left\langle S_{2,0}\right\rangle$ & -0.020 & 0.032 & 0.040 & $0.004 \pm 0.085$ \\
\hline$\left\langle S_{4,0}\right\rangle$ & -0.060 & -0.167 & -0.235 & $-0.311 \pm 0.326$ \\
\hline$\left\langle S_{6,0}\right\rangle$ & 0.020 & 0.023 & 0.055 & $0.990 \pm 1.950$ \\
\hline$\left\langle S_{6,6}\right\rangle$ & -0.013 & -0.013 & -0.061 & $-0.180 \pm 2.300$ \\
\hline
\end{tabular}

${ }^{a}$ Reference 35.

large errors in the experimental results no definite conclusion can be drawn. The effect of temperature is very small. Probably more states need to be included for this kind of calculation.

\section{DISCUSSION AND CONCLUSIONS}

We have presented in this paper a new method that can be used to calculate the properties of orientational disordered (and also ordered) molecular crystals. The states of the crystal are represented in this method as products of onemolecule states. A method that is improved with respect to MF because it includes the orientational pair correlations between the molecules has been used to calculate thermodynamic averages. The states are optimized in order to yield a lowest possible free energy. We have applied the new method to the librational motions of the molecules in the $\beta$ phase of solid nitrogen. Two very important improvements with respect to prior MF calculations have been obtained. A stable disordered phase has been found at all temperatures where $\beta-\mathrm{N}_{2}$ exists, and an $\alpha-\beta$ phase transition has been found at the reasonable temperature $T_{\alpha \beta}=72 \mathrm{~K}$. In this section we will compare our new method with other methods that have been used to calculate properties of orientationally disordered molecular crystals. We will also discuss improvements that can be made and to what other systems the method can be applied.

The method that has been proven to be most useful for the calculations of properties of orientationally disordered molecular crystals is molecular dynamics. ${ }^{2-10}$ The major drawback of this method is that it is a classical method. Therefore, at low temperatures and for light molecules it may give incorrect results because of quantum effects.

The most popular analytical method is a method that is unfortunately also called the MF approximation. ${ }^{38}$ It differs from what we have called MF in the previous sections. Our definition of MF refers to the time-independent Hartree method, whereas the definition of MF in Ref. 38 refers to the 
time-dependent Hartree method (TDH), which we call RPA. ${ }^{39,40}$ Since in Ref. 38 the TDH or RPA method has been written in terms of susceptibilities we shall call this method in what follows the susceptibility approach. It is often used as follows. A free molecule susceptibility is calculated, corresponding to our definition of MF theory (or timeindependent Hartree). The susceptibility for the crystal, corresponding to the TDH or RPA model, is calculated using the free molecule susceptibility and the intermolecular potential. The properties of the crystal can then be calculated from the crystal susceptibility. For example, the orderdisorder phase transition temperature can be determined by looking for soft modes. We want to point out that this is totally incompatible with our present method. The susceptibility approach assumes that the states of the molecules are adapted to the site symmetry; i.e., delocalized states for disordered crystals. ${ }^{13}$ We have shown however, that the states of the molecules in the disordered phase can be localized. When looking for soft modes the susceptibility approach only tests whether these states are stable, but it does not take into account the correlations between the occupations of these states. It implicitly assumes that when the states of the molecules become localized that one of these states becomes predominantly occupied. This means that only certain order-disorder phase transitions can be described by the susceptibility approach. We have shown in a previous paper that soft modes in the time-dependent Hartree approximation indicate an unstable MF state. ${ }^{35}$ This means that the susceptibility approach and MF will yield identical results, because when the susceptibility approach predicts a phase transition then MF should also show that phase transition. Our method, which is an improvement of MF, shows totally different phase transitions.

A method that resembles our method is the pseudo-spin method. One can even look upon our method as a generalized pseudo-spin method. ${ }^{15,16}$ In the pseudo-spin method the exact Hamiltonian is rewritten, making suitable approximations, in the form of the Ising Hamiltonian Eq. (8). Properties are then calculated using one of the approximations that have been devised for the Ising problem. One of the weak points theoretically of the pseudo-spin method is that there is no fixed rule for rewriting the Hamiltonian. Hence, one ends up with a Hamiltonian of the form of Eq. (7). The coefficients and the states of this Hamiltonian have however, not been optimized, but have been obtained in a more or less arbitrary way. Furthermore, the pseudo-spin method is restricted to two states per molecule. Both these deficiencies have been removed in our method.

In order to obtain also quantitatively meaningful results it is necessary to extend the calculations that have been presented in the previous section. The lattice sums have been restricted to only nearest neighbors. It is in principle possible to include more molecules in the lattice sums of Eqs. (28) and (29). In Eq. (28) $z$ then becomes the number of terms in the lattice sums. This would of course lead to a much larger set of equations for the Lagrange multipliers, Eq. (33). How many molecules one can include in the lattice sums depends therefore first of all on the amount of computer time one wants to spend. A much more serious problem is that the problems with negative entropies may become worse. We will show below that these problems arise because some restrictions on the two-particle occupation numbers are missing. The errors that result from neglecting these restrictions may become larger when the lattice sums are extended.

One would also like to include more states per molecule in the calculations. The problem is again that the calculations become larger, now even in two ways. Again the number of Lagrange multipliers Eq. (33) increases, but also the number of equations for the states Eqs. (20), (B4), (B5), and (B6) increases. However, a reduction in the number of states that have to be solved is obtained when from the total number of states only a limited number is allowed to be occupied. The number of Lagrange multipliers in Eq. (33) is only determined by the number of occupied states. The number of equations for the optimization of the states, Eqs. (20), (B4), (B5), and (B6), is still determined by the total number of states, but they simplify appreciably, which can be of help in solving them.

The most important improvement will probably be a better method for obtaining occupation numbers. The only restrictions are given by Eqs. (22) and (23). As was mentioned above, there may be more. This is most easily seen by considering three-particle occupation numbers. These are related to the two-particle occupation numbers via

$$
\left\langle\widehat{E}_{\alpha \alpha}^{(P)} \widehat{E}_{\beta \beta}^{\left(P^{\prime}\right)}\right\rangle_{0}=\sum_{\gamma}\left\langle\widehat{E}_{\alpha \alpha}^{(P)} \widehat{E}_{\beta \beta}^{\left(P^{\prime}\right)} \widehat{E}_{\gamma \gamma}^{\left(P^{\prime \prime}\right)}\right\rangle_{0},
$$

for any $P, P^{\prime}, P^{\prime \prime} \alpha$, and $\beta$. It is easy to see that it is not possible to have three-particle occupation numbers that yield $\left\langle\widehat{E}_{11}^{(P)} \widehat{E}_{11}^{\left(P^{\prime}\right)}\right\rangle_{0}=1,\left\langle\widehat{E}_{11}^{(P)} \widehat{E}_{11}^{\left(P^{\prime \prime}\right)}\right\rangle_{0}=1$, and $\left\langle\widehat{E}_{11}^{\left(P^{\prime}\right)} \widehat{E}_{22}^{\left(P^{\prime \prime}\right)}\right\rangle_{0}$ $=1$. This means that such a combination of two-particle occupation numbers must not arise in the calculations. However, the cluster of two nearest neighbors is simply too small to prevent these inconsistencies. These inconsistencies cause the negative entropies. The cluster variation method can thus be improved by using larger clusters; in particular closed clusters. This will involve three- and more-particle occupation numbers, however, which may complicate the calculations considerably.

An alternative for obtaining the occupation numbers may be the Monte Carlo method. ${ }^{41}$ With this method it is possible in principle to obtain the occupation numbers with any accuracy. There are no fundamental restrictions on the number of states per molecule and the number of molecules in the lattice sums. Furthermore, the method is rather straightforward. There are however two drawbacks. One of them is that Monte Carlo is a very time consuming method. The other is that it is difficult to obtain values for the entropy and hence for the free energy. That means that the method is not suited when we want to study phase transitions like the $\alpha-\beta$ phase transition. Nevertheless, we think that the Monte Carlo method is the most appropriate method for obtaining quantitative results.

We want to comment finally on some projects that may be taken up using the new method that we have presented in this paper. First of all the method might be applied to all the other orientationally disordered molecule crystals. Thereby, 
the improvements mentioned above must be studied. But the method can also be applied to other systems. It would for example be very interesting to use it to study the cooperative Jahn-Teller effect. Some work has already been done in extending the method to fermion and boson systems. Hence, it may be worthwhile to apply the method to solid helium. For finite systems there is no Ising problem as the thermodynamic averages, Eq. (3a), can be obtained by direct calculation of the traces. This means that for example the correlated rotations of methyl groups in organic molecules can be studied advantageously using our method. It may even be possible to extend the method to calculate collective excitations. There is a close relation between the second-order variation of the density operator in the free energy in MF, and the collective excitations as calculated in the time-dependent Hartree approximation. We think that there may be a similar relation between the second-order coefficients of Eq. (B5) and collective excitations.

\section{ACKNOWLEDGMENTS}

I would like to thank Professor Ad van der Avoird, Dr. Wim Briels, and Dr. Paul Wormer for valuable discussions. This investigation was supported in part by the Netherlands Foundation for Chemical Research (S.O.N.) with financial aid from the Netherlands Organization for the Advancement of Pure Research (Z.W.O).

\section{APPENDIX A}

In this Appendix we will prove that the coefficients given by Eq. (15) yield the absolute minimum of $A_{\text {var }}$. We will denote here by $\hat{H}_{0}$ the Hamiltonian of Eq. (7) with the specific coefficients of Eq. (15). The following definition will simplify our notation:

$$
\mathscr{A}\left(\hat{H}_{1} ; \hat{H}_{2}\right) \equiv A_{\hat{H}_{2}}+\left\langle\hat{H}_{1}-\hat{H}_{2}\right\rangle_{\hat{H}_{2}},
$$

with

$$
\begin{aligned}
& A_{\hat{H}_{2}}=-\beta^{-1} \ln Z_{\hat{H}_{1}}, \\
& \langle\hat{H}\rangle_{\hat{H}_{2}}=Z_{\hat{H}_{2}}^{-1} \operatorname{Tr}\left[e^{-\beta \hat{H}_{2}} \widehat{X}\right],
\end{aligned}
$$

and

$$
Z_{\widehat{H}_{2}}=\operatorname{Tr}\left[e^{-\beta \hat{H}_{2}}\right] .
$$

This definition implies that we can write $A_{\text {var }}=\mathscr{A}\left(\hat{H} ; \hat{H}_{0}\right)$.

We now suppose that there are coefficients $c_{P \alpha}^{(1)}$ and $c_{P \alpha, P}^{(2)},{ }^{\prime}$, different from those of Eq. (15), that yield a lower $A_{\text {var }}$. The corresponding Hamiltonian will be denoted by $\hat{H}_{0}^{\prime}$. The basis $\{|\alpha(P)\rangle\}$ of $\hat{H}_{0}^{\prime}$ is the same as that of $\hat{H}_{0}$. Using Eq. (A1) we can write

$$
\mathscr{A}\left(\hat{H} ; \hat{H}_{0}^{\prime}\right)<\mathscr{A}\left(\widehat{H} ; \hat{H}_{0}\right) \text {. }
$$

It is easy to see that

$$
\mathscr{A}\left(\hat{H}_{0} ; \hat{H}_{0}\right)=\mathscr{A}\left(\hat{H} ; \hat{H}_{0}\right)
$$

and

$$
\mathscr{A}\left(\hat{H}_{0} ; \hat{H}_{0}^{\prime}\right)=\mathscr{A}\left(\hat{H} ; \hat{H}_{0}^{\prime}\right)
$$

because the nondiagonal terms of $\hat{H}$ in Eq. (5) vanish. Using Eqs. (A6) and (A7) we obtain

$$
\mathscr{A}\left(\hat{H}_{0} ; \hat{H}_{0}^{\prime}\right)<\mathscr{A}\left(\hat{H}_{0} ; \hat{H}_{0}\right) \text {. }
$$

This however is clearly impossible. We have $\mathscr{A}\left(\hat{H}_{0} ; \hat{H}_{0}\right)$ $=A_{\hat{H}_{0}}$, whereas according to the Gibbs-Bogoliubov inequality Eq. (1) $\mathscr{A}\left(\hat{H}_{0} ; \hat{H}_{0}^{\prime}\right) \geqslant A_{\hat{H}_{0}}$ should hold. This means that Eq. (A5) cannot hold and that consequently the coefficients $c_{P \alpha}^{(1)}$ and $c_{P \alpha, P^{\prime} \beta}^{(2)}$ that are related to the basis $\{|\alpha(P)\rangle\}$ by Eq. (15) yield the absolute minimum of $A_{\text {var }}$.

\section{APPENDIX B}

The derivation of Eq. (20) from Eq. (16) was meant to yield a basis $\{|\alpha(P)\rangle\}$ that minimizes $A_{\text {var }}$. As only $\langle\hat{H}\rangle_{0}$ depends directly on this basis we can also say that we have tried to find the basis that minimizes $\langle\hat{H}\rangle_{0}$. Because the basis must be orthonormal we introduced Lagrange multipliers in Eq. (17). In this Appendix we will introduce new variables for which no restrictions hold. Consequently, we will be able to minimize $\langle\hat{H}\rangle_{0}$ via direct methods. We will show that we can solve Eq. (20) and we will also prove that the solution corresponds to a minimum of $\langle\hat{H}\rangle_{0}$ and $A_{\text {var }}$.

The new variables are the matrix elements of matrices $X^{(P)}$ that are defined by $y^{42,43}$

$$
\mathbf{U}^{(P)}=\exp \left(\mathbf{X}^{(P)}\right) .
$$

Here $\mathrm{U}^{(P)}$ is a matrix with matrix elements $U_{\alpha i}^{(P)}$. The matrix $\mathbf{U}^{(P)}$ in Eq. (B1) is unitary if and only if the matrix $\mathbf{X}^{(P)}$ is anti-Hermitian. We take the lower triangle matrix elements of the $X^{(P)}$ 's as our new unrestricted variables, noting that the diagonal elements are purely imaginary. $\langle\widehat{H}\rangle_{0}$ will thus be interpreted as a function of these variables.

In order to find the minimum of $\langle\hat{H}\rangle_{0}$ we will employ the iterative Newton-Raphson method for finding minima. ${ }^{27}$ In each iteration step $\langle\hat{H}\rangle_{0}$ is expanded as a Taylor series in $\mathbf{X}^{(P)}$ around a trial basis.Cubic and higher order terms are discarded and the remaining quadratic expression is minimized to obtain an improved basis. To be more specific we rewrite $\langle\hat{H}\rangle_{0}$ as in Eq. (13):

$$
\begin{aligned}
\langle\hat{H}\rangle_{0}= & \sum_{P} \sum_{\alpha}\left\langle\widehat{E}_{\alpha \alpha}^{(P)}\right\rangle_{0} \sum_{i j} U_{\alpha i}^{(P) *}\left\langle i(P)\left|\widehat{L}_{P}\right| j(P)\right\rangle U_{\alpha j}^{(P)} \\
& +\frac{1}{2} \sum_{P P} \sum_{\alpha \beta}\left\langle\widehat{E}_{\alpha \alpha}^{(P)} \widehat{E}_{\beta \beta}^{\left(P^{\prime}\right)}\right\rangle_{0} \\
& \times \sum_{i j k l} U_{\alpha i}^{(P)^{*}} U_{\beta k}^{\left(P^{\prime}\right) *}\left\langle i(P) k\left(P^{\prime}\right)\left|\hat{\Phi}_{P P^{\prime}}\right| \hat{\Phi}_{P P^{\prime}}\right. \\
& \times\left|j(P) l\left(P^{\prime}\right)\right\rangle U_{\alpha j}^{(P)} U_{\beta i}^{\left(P^{\prime}\right)} .
\end{aligned}
$$

We identify the reference basis $\{|i(P)\rangle\}$ with our trial basis. The coefficients in the Taylor series, which are the derivatives of $\langle\hat{H}\rangle_{0}$ with respect to $\mathbf{X}^{(P)}$, taken at $\mathbf{X}^{(P)}=\mathbf{0}$, are most easily calculated by first determining the derivatives of $U_{a i}^{(P)}$ with respect to the $X_{i j}^{(P)}$ 's. We find

$$
\begin{aligned}
& \left(\frac{\partial U_{\alpha i}^{(P)}}{\partial X_{j k}^{\left(P^{\prime}\right)}}\right)_{\mathbf{x}=0}=\left(\frac{\partial U_{\alpha i}^{(P)^{*}}}{\partial X_{j k}^{\left(P^{\prime}\right)^{*}}}\right)_{\mathrm{x}=\mathbf{0}}=\delta_{P P^{\prime}} \delta_{\alpha j} \delta_{i k}, \\
& \left(\frac{\partial U_{a i}^{(P)}}{\partial X_{j k}^{\left(P^{\prime}\right) *}}\right)_{\mathbf{x}=\mathbf{0}}=\left(\frac{\partial U_{\alpha i}^{(P)^{*}}}{\partial X_{j k}^{\left(P^{\prime}\right)}}\right)_{\mathbf{X}=\mathbf{0}}=-\delta_{P P^{\prime}} \delta_{\alpha k} \delta_{i j},
\end{aligned}
$$




$$
\begin{aligned}
& \left(\frac{\partial^{2} U_{\alpha i}^{(P)}}{\partial X_{j k}^{\left(P^{\prime}\right)} \partial X_{l m}^{\left(P^{-1}\right)}}\right)_{\mathrm{x}=0} \\
& =\left(\frac{\partial^{2} U_{\alpha i}^{(P)^{*}}}{\partial X_{j k}^{\left(P^{\prime}\right) *} \partial X_{l m}^{\left(P^{*}\right)^{*}}}\right)_{\mathbf{x}=\mathbf{0}} \\
& =\frac{1}{2} \delta_{P P^{\prime}} \delta_{P P^{*}}\left(\delta_{\alpha j} \delta_{i m} \delta_{k l}+\delta_{\alpha l} \delta_{i k} \delta_{j m}\right) \text {, } \\
& \left(\frac{\partial^{2} U_{\alpha i}^{(P)}}{\partial X_{j k}^{\left(P^{\prime}\right)} \partial X_{l m}^{\left(P^{*}\right)^{*}}}\right)_{\mathbf{x}=\mathbf{0}} \\
& =\left(\frac{\partial^{2} U_{\alpha i}^{(P)^{*}}}{\partial X_{j k}^{\left(P^{\prime}\right) *} \partial X_{l m}^{\left(P^{*}\right)}}\right)_{\mathbf{X}=\mathbf{0}} \\
& =-\frac{1}{2} \delta_{P P} \delta_{P P} \cdot\left(\delta_{\alpha j} \delta_{i l} \delta_{k m}+\delta_{\alpha m} \delta_{i k} \delta_{j l}\right) \text {, } \\
& \left(\frac{\partial^{2} U_{\alpha i}^{(P)}}{\partial X_{j k}^{\left(P^{\prime}\right) *} \partial X_{l m}^{\left(P^{*}\right) *}}\right)_{\mathrm{X}=\mathbf{0}}
\end{aligned}
$$

$$
\begin{aligned}
& =\left(\frac{\partial^{2} U_{\alpha i}^{(P)^{*}}}{\partial X_{j k}^{\left(P^{\prime}\right)} \partial X_{l m}^{\left(P^{*}\right)}}\right)_{\mathrm{x}=0} \\
& =\frac{1}{2} \delta_{P P^{\prime}} \delta_{P P^{*}}\left(\delta_{\alpha k} \delta_{i l} \delta_{j m}+\delta_{\alpha m} \delta_{i j} \delta_{k l}\right),
\end{aligned}
$$

where for all $X_{i j}^{(P)} i \geqslant j$ holds. The calculation of the derivatives of $\langle\hat{H}\rangle_{0}$ is lengthy but straightforward. We find for the first derivatives

$$
\begin{aligned}
\left(\frac{\partial\langle\hat{H}\rangle_{0}}{\partial X_{i j}^{(P)}}\right)_{\mathbf{x}=0}= & \left(\frac{\partial\langle\hat{H}\rangle_{0}}{\partial X_{i j}^{(P)^{*}}}\right)_{\mathbf{x}=0}^{*} \\
= & \left\langle i(P)\left|\hat{L}_{P}\right| j(P)\right\rangle\left[\left\langle\widehat{E}_{i}^{(P)}\right\rangle_{0}-\left\langle\widehat{E}_{j j}^{(P)}\right\rangle_{0}\right] \\
& +\sum_{Q} \sum_{k}\left\langle i(P) k(Q)\left|\hat{\Phi}_{P Q}\right| j(P) k(Q)\right\rangle \\
& \times\left[\left\langle\widehat{E}_{i i}^{(P)} \widehat{E}_{k k}^{(Q)}\right\rangle_{0}-\left\langle\widehat{E}_{i j}^{(P)} \hat{E}_{k k}^{(Q)}\right\rangle_{0}\right] .
\end{aligned}
$$

We note the resemblance to Eq. (20). The second derivatives are given by

$$
\begin{aligned}
& \left(\frac{\partial^{2}\langle\hat{H}\rangle_{0}}{\partial X_{i j}^{(P)} \partial X_{k l}^{\left(P^{\prime}\right)}}\right)_{\mathbf{x}=0}=\left(\frac{\partial^{2}\langle\hat{H}\rangle_{0}}{\partial X_{i j}^{(P)^{*}} \partial X_{k l}^{\left(P^{\prime}\right)^{*}}}\right)_{\mathrm{X}=\mathbf{0}}^{*} \\
& =\frac{1}{2} \delta_{P P^{\prime}}\left[\delta_{j k}\left\langle i(P)\left|\hat{L}_{P}\right| l(P)\right\rangle-\delta_{i l}\left\langle k(P)\left|\hat{L}_{P}\right| j(P)\right\rangle\right]\left[\left\langle\widehat{E}_{i i}^{(P)}\right\rangle_{0}-\left\langle\hat{E}_{j j}^{(P)}\right\rangle_{0}-\left\langle\hat{E}_{k k}^{(P)}\right\rangle_{0}+\left\langle\hat{E}_{l l}^{(P)}\right\rangle_{0}\right] \\
& +\frac{1}{2} \delta_{P P^{\prime}} \sum_{Q} \sum_{m}\left[\delta_{j k}\left\langle i(P) m(Q)\left|\hat{\Phi}_{P Q}\right| l(P) m(Q)\right\rangle-\delta_{i l}\left\langle k(P) m(Q)\left|\hat{\Phi}_{P Q}\right| j(P) m(Q)\right\rangle\right] \\
& \times\left[\left\langle\widehat{E}_{i i}^{(P)} \widehat{E}_{m m}^{(Q)}\right\rangle_{0}-\left\langle\widehat{E}_{j j}^{(P)} \widehat{E}_{m m}^{(Q)}\right\rangle_{0}-\left\langle\widehat{E}_{k k}^{(P)} \widehat{E}_{m m}^{(Q)}\right\rangle_{0}+\left\langle\widehat{E}_{l i}^{(P)} \widehat{E}_{m m}^{(Q)}\right\rangle_{0}\right] \\
& +\left\langle i(P) k\left(P^{\prime}\right)\left|\hat{\Phi}_{P P^{\prime}}\right| j(P) l\left(P^{\prime}\right)\right\rangle \\
& \times\left[\left\langle\widehat{E}_{i i}^{(P)} \widehat{E}_{k k}^{\left(P^{\prime}\right)}\right\rangle_{0}+\left\langle\widehat{E}_{j j}^{(P)} \widehat{E}_{l i}^{\left(P^{\prime}\right)}\right\rangle_{0}-\left\langle\widehat{E}_{i i}^{(P)} \widehat{E}_{l i}^{\left(P^{\prime}\right)}\right\rangle_{0}-\left\langle\widehat{E}_{j j}^{(P)} \widehat{E}_{k k}^{\left(P^{\prime}\right)}\right\rangle_{0}\right],
\end{aligned}
$$

and

$$
\begin{aligned}
& \left(\frac{\partial^{2}\langle\hat{H}\rangle_{0}}{\partial X_{i j}^{(P)} \partial X_{k l}^{\left(P^{\prime}\right)^{*}}}\right)_{\mathbf{x}=0}=\left(\frac{\partial^{2}\langle\hat{H}\rangle_{0}}{\partial X_{i j}^{(P)^{*}} \partial X_{k l}^{\left(P^{\prime}\right)}}\right)_{\mathrm{x}=\mathbf{0}}^{*} \\
& =-\frac{1}{2} \delta_{P P^{\prime}}\left[\delta_{j l}\left\langle i(P)\left|\hat{L}_{P}\right| k(P)\right\rangle-\delta_{i k}\left(l(P)\left|\hat{L}_{P}\right| j(P)\right\rangle\right]\left[\left\langle\widehat{E}_{i i}^{(P)}\right\rangle_{0}-\left\langle\widehat{E}_{j j}^{(P)}\right\rangle_{0}+\left\langle\widehat{E}_{k k}^{(P)}\right\rangle_{0}-\left\langle\widehat{E}_{i l}^{(P)}\right\rangle_{0}\right] \\
& -\frac{1}{2} \delta_{P P^{\prime}} \sum_{Q} \sum_{m}\left[\delta_{j l}\left\langle i(P) m(Q)\left|\hat{\Phi}_{P Q}\right| k(P) m(Q)\right\rangle-\delta_{i k}\left\langle l(P) m(Q)\left|\hat{\Phi}_{P Q}\right| j(P) m(Q)\right\rangle\right] \\
& \times\left[\left\langle\widehat{E}_{i i}^{(P)} \widehat{E}_{m m}^{(Q)}\right\rangle_{0}-\left\langle\widehat{E}_{j j}^{(P)} \widehat{E}_{m m}^{(Q)}\right\rangle_{0}+\left\langle\widehat{E}_{k k}^{(P)} \widehat{E}_{m m}^{(Q)}\right\rangle_{0}-\left\langle\widehat{E}_{l i}^{(P)} \widehat{E}_{m m}^{(Q)}\right\rangle_{0}\right] \\
& +\left\langle i(P) l\left(P^{\prime}\right)\left|\widehat{\Phi}_{P P^{\prime}}\right| j(P) k\left(P^{\prime}\right)\right\rangle \\
& \times\left[\left\langle\widehat{E}_{i i}^{(P)} \widehat{E}_{k k}^{\left(P^{\prime}\right)}\right\rangle_{0}+\left\langle\widehat{E}_{j j}^{(P)} \widehat{E}_{i l}^{\left(P^{\prime}\right)}\right\rangle_{0}-\left\langle\widehat{E}_{i i}^{(P)} \widehat{E}_{i l}^{\left(P^{\prime}\right)}\right\rangle_{0}-\left\langle\widehat{E}_{j j}^{(P)} \widehat{E}_{k k}^{\left(P^{\prime}\right)}\right\rangle_{0}\right] .
\end{aligned}
$$

The following set of linear equations has to be solved in each iteration step:

$$
\begin{gathered}
\left(\frac{\partial\langle\hat{H}\rangle_{0}}{\partial X_{i j}^{(P)}}\right)_{\mathbf{x}=0}+\sum_{P^{\prime}} \sum_{k l}\left[X_{k l}^{\left(P^{\prime}\right)}\left(\frac{\partial^{2}\langle\hat{H}\rangle_{0}}{\partial X_{i j}^{(P)} \partial X_{k l}^{\left(P^{\prime}\right)}}\right)_{\mathbf{X}=0}\right. \\
\left.+X_{k l}^{\left(P^{\prime}\right) *}\left(\frac{\partial^{2}\langle\hat{H}\rangle_{0}}{\partial X_{i j}^{(P)^{*}} \partial X_{k l}^{\left(P^{\prime}\right)}}\right)_{\mathbf{x}=0}\right]=0
\end{gathered}
$$

(B6a)

$$
\begin{aligned}
& \left(\frac{\partial\langle\hat{H}\rangle_{0}}{\partial X_{i j}^{(P)^{*}}}\right)_{\mathbf{X}=0}+\sum_{P^{\prime}} \sum_{k l}\left[X_{k l}^{\left(P^{\prime}\right)}\left(\frac{\partial^{2}\langle\hat{H}\rangle_{0}}{\partial X_{i j}^{(P)} \partial X_{k l}^{\left(P^{\prime}\right)}}\right)_{\mathbf{X}=0}\right. \\
& \left.+X_{k l}^{\left(P^{\prime}\right)^{*}}\left(\frac{\partial^{2}\langle\hat{H}\rangle_{0}}{\partial X_{i j}^{(P)^{*}} \partial X_{k l}^{\left(P^{\prime}\right)^{*}}}\right)_{\mathbf{X}=\mathbf{0}}\right]=0,
\end{aligned}
$$

Convergence of the Newton-Raphson method is reached in the $n$th iteration step when for the solution $\mathbf{X}_{n}^{(P)}$ of this equation in the $n$th step $\mathbf{X}_{n}^{(P)}=\mathbf{0}$ holds. This condition can only 
be fulfilled when the first derivatives in Eq. (B6) become zero. Hence, the Newton-Raphson method yields a solution of Eq. (20). Furthermore, the second derivatives in Eq. (B6) must form a positive definite matrix, because the solution must correspond to a minimum of $\langle\hat{H}\rangle_{0}$. The second derivatives thus yield a test that we can use to determine whether we have really found a minimum for $A_{\text {var }}$.

We want finally to comment on how to obtain the unitary matrices $\mathbf{U}^{(P)}$ from the anti-Hermitian matrices $\mathbf{X}_{n}^{(P)}$. The matrices $\mathbf{i} \mathbf{X}_{n}^{(P)}$ are Hermitian and can thus be diagonalized. This means that $\mathbf{X}_{n}^{(P)}$. can also be diagonalized. We define the unitary matrices $V_{n}^{(P)}$ and the diagonal matrices $\mathbf{\Lambda}_{n}^{(P)}$ by

$$
\mathbf{X}_{n}^{(P)} \mathbf{V}_{n}^{(P)}=\mathbf{V}_{n}^{(P)} \Lambda_{n}^{(P)}
$$

The diagonal elements of $\Lambda_{n}^{(P)}$ are purely imaginary. The matrices $U_{n}^{(P)}$ can be defined by

$$
\mathbf{U}_{n}^{(P)}=\mathbf{V}_{n}^{(P)} \exp \left(\mathbf{\Lambda}_{n}^{(P)}\right) \mathbf{V}_{n}^{(P) \dagger} \text {. }
$$

The matrices $U^{(P)}$ are then given by

$$
\mathbf{U}^{(P)}=\mathbf{U}_{n^{\prime}}^{(P)} \cdots \mathbf{U}_{2}^{(P)} \mathbf{U}_{1}^{(P)},
$$

where $n^{\prime}$ denotes the step in which convergence has been reached.

'J. van Kranendonk, Solid Hydrogen (Plenum, New York, 1983).

${ }^{2}$ M. L. Klein, D. Lévesque, and J.-J. Weis, J. Chem. Phys. 74, 2566 (1981).

${ }^{3}$ M. L. Klein and J.-J. Weis, J. Chem. Phys. 67, 217 (1977).

${ }^{4}$ M. L. Klein and I. R. McDonald, J. Chem. Phys. 79, 2333 (1983).

${ }^{5}$ M. Ferrario, I. R. McDonald, and M. L. Klein, J. Chem. Phys. 84, 3975 (1986).

${ }^{6}$ R. W. Impey, M. Sprik, and M. L. Klein, J. Chem. Phys. 83, 3638 (1985).

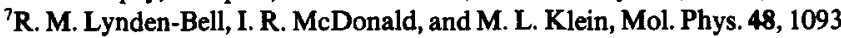
(1983).

${ }^{8}$ R. M. Lynden-Bell, M. L. Klein, and I. R.McDonald, Z. Phys. B 54, 325 (1984).

${ }^{9}$ M. T. Dove and R. M. Lynden-Bell, J. Phys. C 19, 3343 (1986).

${ }^{10}$ M. T. Dove, J. Phys. C 19, 3325 (1986).

${ }^{11}$ K. H. Michel and J. Naudts, J. Chem. Phys. 67, 547 (1977).

${ }^{12}$ K. H. Michel, J. Chem. Phys. 84, 3451 (1986).

${ }^{13}$ K. H. Michel and K. Parlinski, Phys. Rev. B 31, 1823 (1985).
${ }^{14}$ K. H. Michel and J. M. Rowe, Phys. Rev. B 32, 5818, 5827 (1985).

${ }^{15}$ M. Tokunaga and T. Matsubara, Prog. Theor. Phys. 35, 581 (1966).

${ }^{16}$ T. Luty and R. W. Munn, J.Chem. Phys. 80, 3321 (1984).

${ }^{17}$ C. Domb, Adv. Phys. 9, 149 (1960).

${ }^{18}$ H. L. Friedman, A Course in Statistical Mechanics (Prentice-Hall, London, 1985).

${ }^{19}$ J. G. Kirkwood, J. Chem. Phys. 8, 205 (1940).

${ }^{20}$ H. M. James and T. A. Keenan, J. Chem. Phys. 31, 12 (1959).

${ }^{21}$ N. R. Werthamer, in Rare Gas Solids, edited by M. L. Klein and J. Venables (Academic, London, 1976), Vol. I.

${ }^{22}$ R. J. Baxter, Exactly Solved Models in Statistical Physics (Academic, London, 1982).

${ }^{23}$ Phase Transitions and Critical Phenomena, edited by C. Domb and M. S. Green (Academic, London, 1976).

${ }^{24}$ R. Kikuchi, Phys. Rev. 81, 988 (1951).

${ }^{25}$ M. Kurata and R. Kikuchi, J. Chem. Phys. 21, 434 (1953).

${ }^{26} \mathrm{D}$. M. Burley, in Phase Transitions and Critical Phenomena, edited by C. Domb and M. S. Green (Academic, London, 1976), Vol. 2.

${ }^{27}$ R. F. Churchhouse, Numerical Methods, Vol. III of Handbook of Applicable Mathematics, edited by W. Ledermann (Wiley, Chichester, 1981).

${ }^{28}$ S. Buchsbaum, R. L. Mills, and D. Schiferl, J. Phys. Chem. 88, 2522 (1984).

${ }^{29}$ W. E. Streib, T. H. Jordan, and W. N. Lipscomb, J. Chem. Phys. 37, 2962 (1962).

${ }^{30}$ T. H. Jordan, H. W. Smith, W. E. Streib, and W. N. Lipscomb, J. Chem. Phys. 41, 756 (1964).

${ }^{31}$ A. F. Schuch and R. L. Mills, J. Chem. Phys. 52, 6000 (1970).

${ }^{32}$ B. M. Powell, G. Dolling, and H. F. Nieman, J. Chem. Phys. 79, 982 (1983).

${ }^{33}$ A. S. deReggi, P. C.Canepa, and T. A. Scott, J. Magn. Reson. 1, 144 (1969).

${ }^{34}$ J. C. Raich, N. S.Gillis, and T. R. Koehler, J. Chem. Phys. 61, 1411 (1974).

${ }^{35}$ A. van der Avoird, W. J. Briels, and A. P. J. Jansen, J. Chem. Phys. 81, 3658 (1984).

${ }^{36}$ R. M. Berns and A. van der Avoird, J. Chem. Phys. 72, 6107 (1980).

${ }^{37}$ A. van der Avoird, P. E. S. Wormer, and A. P. J. Jansen, J. Chem. Phys. 84, 1629 (1986).

${ }^{38}$ J. C. Raich, H. Yasuda, and E. R. Bernstein, J. Chem. Phys. 78, 6209 (1983).

${ }^{39}$ W. J. Briels, A. P. J. Jansen, and A. van der Avoird, J. Chem. Phys. 81, 4118 (1984).

${ }^{40}$ A. P. J. Jansen and A. van der Avoird, J. Chem. Phys. 86, 3583 (1987).

${ }^{41} \mathrm{~K}$. Binder, in Monte Carlo Methods in Statistical Physics, edited by K. Binder (Springer, Berlin, 1979).

${ }^{42}$ R. McWeeny, Symp. Faraday Soc. 2, 7 (1968).

${ }^{43} \mathrm{~J}$. Douady, Y. Ellinger, R. Subra, and B. Levy, Comp. Phys. Commun. 17, 23 (1979). 